\title{
Treatment with a polymyxin B filter to capture endotoxin in sepsis patients: is there a signal for therapeutic efficacy?
}

\author{
Peter Pickkers ${ }^{1,2^{*}}$ (D) and James A. Russell ${ }^{3}$
}

(C) 2018 Springer-Verlag GmbH Germany, part of Springer Nature

The discovery of endotoxin, a key component of the membrane of Gram negative bacteria, as one of the main pathogen-associated molecular patterns associated with impaired clinical outcome [1] prompted the hypothesis that 'blood purification' might be of clinical benefit for patients with sepsis. Polystyrene fiber filters coated with polymyxin B bind endotoxin avidly. Apart from many case series (summarized in a meta-analysis of trials primarily from Japanese origin, suggesting a survival benefit for patients treated with polymyxin B hemoperfusion [2]), only two prospective randomized controlled clinical trials (RCTs) were conducted. Both trials had shortcomings. The EUPHAS trial [3] was small $(n=64)$ and early termination increased the risk of type I errors [4], and while the ABDOMIX RCT $(n=232)$ [5] was open-label, disease severity and overall mortality were moderate and incomplete PMX treatment sessions occurred in $11 \%$ of the patients. Plasma endotoxin levels were not measured in either RCT.

Recently, the results of the Euphrates RCT were published [6]. This RCT has the largest recruiting number so far $(n=244)$ and arguably the highest scientific quality, including unique features such as quantifiable patient enrichment by use of the Endotoxin Activity Assay (EAA) as a predictive biomarker to confirm endotoxemia (defined as EAA > 0.6) and use of a detailed sham-procedure as a treatment-blinding mechanism. Unfortunately, the trial did not show a survival benefit in the patients treated with the PMX-filter compared to the shamgroup. In the current issue of Intensive Care Medicine

\footnotetext{
${ }^{*}$ Correspondence: peter.pickkers@radboudumc.nl

${ }^{2}$ Radboud Center for Infectious diseases, Nijmegen, The Netherlands

Full author information is available at the end of the article
}

[7], the investigators of the Euphrates trial report their exploratory post-hoc analysis that focuses on a specific endotoxin level-defined subgroup of patients. There is a therapeutic survival effect of the PMX-filter in patients with an EAA 0.06-0.89 $(n=194)$, while there was no benefit in patients with an EAA $>0.9(n=50)$ in an adjusted analysis. The authors suggest that the lack of therapeutic benefit in patients who had EAA $>0.9$ was due to saturation of the PMX filter with endotoxin or because these patients have had such high levels of endotoxin that their prognosis is not improved by the PMX intervention.

It could be worthwhile to investigate whether or not there are more responsive subgroups of patients who might benefit from the treatment, especially when there is a lack of overall benefit in a large trial. Therefore, we applaud every effort to find value and understand heterogeneous treatment effects in trials with negative primary outcomes. It is unfortunate however, that saturation of the PMX-filter in patients with high circulating endotoxin levels was not considered prior to the trial, especially when only $2 \mathrm{~h}$ of treatment with the filter per day was applied and more intense treatment (i.e. more filter changes or longer duration of application of the filter per session) would have been feasible.

Several comments are relevant to adequately interpret this exploratory post-hoc analysis. First, the primary outcome analysis of RCTs, including its post-hoc analyses, should be an unadjusted analysis; adjustments for a selection of variables is normally not done because other (non-measured) variables might also not be balanced between treatment groups and one cannot correct for these with adjusted analyses. However, in their primary analysis, these investigators adjusted for two key baseline variables, mean arterial blood pressure

\section{Springer}


and disease severity (APACHE II) scores, both of which were not significantly different between groups at baseline. Importantly, 28-day mortality was not significantly different between PMX vs. sham treatment in the unadjusted analysis of this EAA 0.6-0.89 subgroup. To be clear, the unadjusted analysis - the correct analysis in an RCT to determine the primary result-showed no benefit of PMX purification in the EAA 0.6-0.89 subgroup compared to the sham-group. Second, the hypothesis of this post-hoc analysis was that the subgroup of patients with EAA 0.6-0.9 are in the 'addressable range', i.e. a not extremely elevated endotoxin level that is susceptible to the endotoxin lowering effect of the PMX-filter. Overall, lower endotoxin levels were associated with higher survival; however, the decrease in EAA over time was not different between the PMX and sham groups. Several explanations, including recompartmentalization of endotoxin, are possible but this lack of more pronounced EAA lowering by PMX does not strengthen the biological plausibility of the hypothesis that increasing endotoxin clearance increases survival. Finally, the EAA-range used in this sub-study has arbitrary cut-offs; it would have been preferable to demonstrate the relevance of the baseline endotoxin concentration in a multivariable analysis, using PMX-treatment and EAA as covariates and survival as outcome measure and then demonstrate whether or not treatment with the PMX-filter still contributes to a statistically significant survival benefit.

In conclusion, endotoxin, one of the most potent mediators of Gram-negative sepsis, is found in high levels in approximately half of patients with septic shock. Polymyxin B binds endotoxin, and although in theory blood "purification" to remove endotoxin in sepsis is a valid approach, sound evidence that this treatment translates to clinical benefit for sepsis patients is lacking. The current result of treatment with a PMX-filter harkens back to endotoxin antibody treatments that did not increase survival [8]. The current exploratory post-hoc analysis is of interest, but we emphasize that exploratory post-hoc analyses do not prove therapeutic efficacy, but rather are hypothesis-generating. There is increased risk of bias and not using correction for multiple testing in posthoc analyses further increases the risk of false-positive results. This means that we should be extremely careful with the interpretation of these analyses and that clinical efficacy indeed needs to be determined prospectively in randomized controlled trials.

\section{Author details}

${ }^{1}$ Department of Intensive Care Medicine (710), Radboud University Medical Centre, Geert Grooteplein Zuid 10, P.O. Box 9101, 6500 HB Nijmegen, The Netherlands. ${ }^{2}$ Radboud Center for Infectious diseases, Nijmegen, The Netherlands. ${ }^{3}$ Centre for Heart Lung Innovation, and Division of Critical Care Medicine, St. Paul's Hospital and University of British Columbia, 1081 Burrard St., Vancouver, BC V6Z 1Y6, Canada.

\section{Conflicts of interest}

Dr. Pickkers has received honoraria from Gambro-Baxter. Dr. Russell reports patents owned by the University of British Columbia (UBC) that are related to the use of PCSK9 inhibitor(s) in sepsis and related to the use of vasopressin in septic shock. Dr. Russell is an inventor on these patents. Dr. Russell was a founder, Director and shareholder in Cyon Therapeutics Inc. Dr. Russell is a shareholder in Molecular You Corp. Dr. Russell reports receiving consulting fees in the last 3 years from: (1) Asahi Kesai Pharmaceuticals of America (AKPA) (developing recombinant thrombomodulin in sepsis). (2) SIB Therapeutics LLC (developing a sepsis drug). No longer actively consulting for the following: (3) La Jolla Pharmaceuticals (developing angiotensin II; Dr. Russell chaired the DSMB of a trial of angiotensin II from 2015 to 2017) - no longer actively consulting. (4) Ferring Pharmaceuticals (manufactures vasopressin and was developing selepressin)—no longer actively consulting. (5) Cubist Pharmaceuticals (now owned by Merck; formerly was Trius Pharmaceuticals; developing antibiotics)—no longer actively consulting. (6) Grifols (sells albumin)—no longer actively consulting. (7) CytoVale Inc. (developing a sepsis diagnostic)—no longer actively consulting. Dr. Russell reports having received an investigatorinitiated grant from Grifols (entitled "Is HBP a mechanism of albumin's efficacy in human septic shock?") that was provided to and administered by UBC.

Received: 16 November 2018 Accepted: 21 November 2018 Published online: 3 December 2018

\section{References}

1. Opal SM et al (1999) Relationship between plasma levels of lipopolysaccharide (LPS) and LPS-binding protein in patients with severe sepsis and septic shock. J Infect Dis 180(5):1584-1589

2. Zhou F et al (2013) Blood purification and mortality in sepsis: a metaanalysis of randomized trials. Crit Care Med 41 (9):2209-2220

3. Cruz DN et al (2009) Early use of polymyxin B hemoperfusion in abdominal septic shock: the EUPHAS randomized controlled trial. JAMA 301(23):2445-2452

4. Vincent JL (2009) Polymyxin B hemoperfusion and mortality in abdominal septic shock. JAMA 302(18):1968 (author reply 1969-70)

5. Payen DM et al (2015) Early use of polymyxin B hemoperfusion in patients with septic shock due to peritonitis: a multicenter randomized control trial. Intensive Care Med 41 (6):975-984

6. Dellinger $R$ et al (2018) Effect of targeted polymyxin B hemoperfusion on 28-day mortality in patients with septic shock and elevated endotoxin level: the euphrates randomized clinical trial. JAMA 320(14):1455-1463

7. Klein DJ, Foster D, Walker PM, Bagshaw SM, Mekonnen H, Antonelli M (2018) Polymyxin B hemoperfusion in endotoxemic septic shock patients without extreme endotoxemia: a post hoc analysis of the EUPHRATES trial. ICM. https://doi.org/10.1007/s00134-018-5463-7

8. Opal SM et al (2013) Effect of eritoran, an antagonist of md2-tlr4, on mortality in patients with severe sepsis: the access randomized trial. JAMA 309(11):1154-1162 